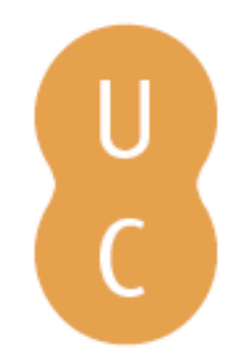

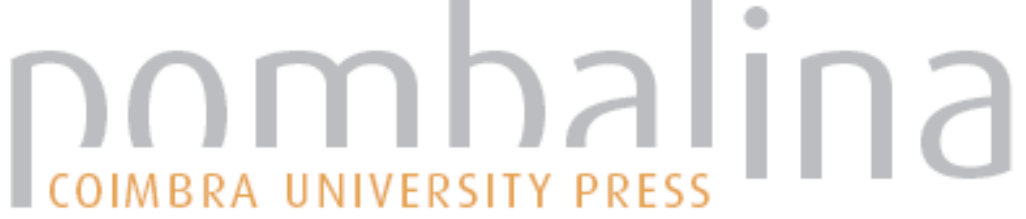

\section{Il cibo come metafora politica nelle ad familiares di Cicerone}

\author{
Autor(es): $\quad$ Ginelli, Francesco
}

Publicado por: Imprensa da Universidade de Coimbra; Annablume

URL

persistente: $\quad$ URI:http://hdl.handle.net/10316.2/39616

DOI: $\quad$ DOl:https://doi.org/10.14195/978-989-26-1191-4_7

Accessed : $\quad$ 26-Apr-2023 15:10:21

A navegação consulta e descarregamento dos títulos inseridos nas Bibliotecas Digitais UC Digitalis, UC Pombalina e UC Impactum, pressupõem a aceitação plena e sem reservas dos Termos e Condições de Uso destas Bibliotecas Digitais, disponíveis em https://digitalis.uc.pt/pt-pt/termos.

Conforme exposto nos referidos Termos e Condições de Uso, o descarregamento de títulos de acesso restrito requer uma licença válida de autorização devendo o utilizador aceder ao(s) documento(s) a partir de um endereço de IP da instituição detentora da supramencionada licença.

Ao utilizador é apenas permitido o descarregamento para uso pessoal, pelo que o emprego do(s) título(s) descarregado(s) para outro fim, designadamente comercial, carece de autorização do respetivo autor ou editor da obra.

Na medida em que todas as obras da UC Digitalis se encontram protegidas pelo Código do Direito de Autor e Direitos Conexos e demais legislação aplicável, toda a cópia, parcial ou total, deste documento, nos casos em que é legalmente admitida, deverá conter ou fazer-se acompanhar por este aviso.

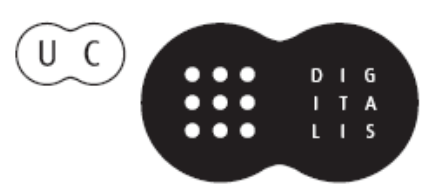




\section{IL CIBO COME METAFORA POLITICA NELLE Ad FAMiliares di Cicerone Food as political metaphor in Cicero's Ad familiares}

Francesco Ginelli

Università degli Studi di Verona

Dipartimento di Filologia, Letteratura e Linguistica

francesco.ginell@unirer.it

Accenni al cibo sono diffusi in tutta l'opera ciceroniana. Qui verrà analizzato un particolare valore presente nelle ad familiares: la metafora politica. Il collegamento di una precisa pietanza con un determinato personaggio viene utilizzato per prendersi gioco di quest'ultimo. In Fam. 7, 26 Cicerone descrive fortissimi disturbi intestinali causatigli da una cena le cui portate erano, in rispetto alla legge suntuaria di Cesare, a base di bietole e malva: l'episodio offre lo spunto per criticare tale provvedimento che, invece di regolare gli eccessi limitando il consumo di carni, creerebbe solo dolori di stomaco. Fam. 9, 18 gioca sull'ambiguità di pavo, animale allora pregiato ma ritenuto simbolo di vanità, e su ius, "diritto", ma anche "brodo": i cesariani Irzio e Dolabella sarebbero pavoni che sguazzano, ignari, nel brodo preparato da Cicerone, loro maestro di retorica. La parsimonia della tavola di Peto in Fam. 9, 16 e 9, 18 è un'occasione per criticare la soluzione dei debiti adottata da Cesare. Un caso particolare è Fam. 9, 24:1'aruspice Spurinna, che intimò a Cesare di non recarsi in senato alle Idi di Marzo, inviterebbe Peto, con amaro capovolgimento ironico, a frequentare nuovamente i banchetti degli amici.

Keywords: Cicerone, Epistole, Cibo, Metafora, Politica

Food references are scattered in all Cicero's works. This paper will analyze one particular meaning in the ad familiares: the political metaphor. The connection of a specific food with a particular person is employed to make a joke against him. In Fam. 7, 26, Cicero describes heavy digestion problems caused by a dinner with chard and mallow, in accordance with Caesar's recent sumptuary law: the episode offers the opportunity to critique this rule that, instead of regulating meat intake, would cause only stomachache. Fam. 9, 18 plays with the ambiguity of pavo, a very precious animal but also a symbol of vanity, and ius, "law", but also "broth": the two Caesarians, Hirtius and Dolabella, would be two unaware peacocks that wallows in the soup cooked by Cicero, their rhetoric master. The frugality of Peto's table in Fam. 9, 16 and 9, 18 is a way to disapprove Caesar's solution of debts. Fam. 9, 24 represents a particular case: the haruspex Spurinna, who warned Caesar not to go in senate on the Ides of March, would exhort Peto, with a bitter irony, to go outside for having dinner with a friend as in the past.

Keywords: Cicero, Letters, Food, Metaphor, Politics 
I corpora epistolari ciceroniani contano numerosi riferimenti a cene e banchetti. Rapidi accenni e approfondite descrizioni mostrano che spesso queste occasioni non erano semplici momenti di convivialità, ma spazi sociali in cui stringere relazioni e rinsaldare amicizie ${ }^{1}$. A questi racconti si affiancano anche le descrizioni di cibi e pietanze che venivano di volta in volta consumati dai commensali: riferimenti a carni, insaccati, molluschi, ortaggi, condimenti e vini hanno un grande valore documentario per capire pratiche e abitudini della società descritta da Cicerone. Tuttavia nella raccolta delle ad familiares si può notare che, parallelamente alla semplice dimensione materiale, emerge un diverso valore legato al cibo: la metafora politica. Sebbene in tale silloge epistolare non manchino giochi di parole legati all'uso traslato di particolari termini ${ }^{2}$ oppure allusioni ironiche ai presunti poteri di specifici alimenti ${ }^{3}$, in determinati passi è possibile notare come Cicerone carichi di un duplice significato alcuni cibi, creando così argute metafore al fine di ironizzare contro avversari politici e divertire il proprio destinatario. Scopo del presente elaborato sarà pertanto quello di indagare questo specifico contesto, illustrandone i meccanismi di funzionamento e i referenti cui di volta in volta si allude.

\section{I) Malva, bietola e leggi sul lusso.}

In Cic. Fam. 7, 26 Cicerone scrive all'amico Fabio Gallo 4 , ricco uomo d'affari con interessi epicurei ${ }^{5}$, di essere appena guarito da forti disturbi

\footnotetext{
${ }^{1}$ Per un'analisi del valore sociale e politico della cena nella Roma di Cicerone cfr. Tabacco 2009.

${ }^{2}$ Cfr. lo scherzo col verbo sapere in Cic. Fam. 1, 10: istic ubi solus sapere videare, dove se usato con valore concreto ha il significato di "avere sapore", se traslato quello di "essere assennato". Cicerone se ne serve sempre per ironizzare sul sapere giuridico-legale, cfr. Shackleton Bailey 1977a: 318 e 333: il gioco di parole, ripreso anche in Fam. 7, 10, 1 e indirizzato al giovane Trebazio Testa, trova diffusione nella letteratura latina già da Enn. scen. 273 Vahlen $^{2}=221$ Jocelyn: qui ipse sibi sapiens prodesse non quit, nequiquam sapit, verso tramandato proprio dallo stesso Cicerone in Fam. 7, 6, 2 (anche questa indirizzata al già citato Trebazio).

${ }^{3}$ In Cic. Fam. 7, 20, 1 Cicerone ironizza con il solito Trebazio sui poteri magici del fiore di loto (sulla tradizione del testo cfr. Garbarino-Tabacco 2008b: 314, n. 7). Préchac 1913 ha interpretato il passo sostenendo che l'oratore, nel tentativo di convincere il giovane giurista a non abbandonare la villa di famiglia a Velia, gli comunicherebbe che la pianta di loto presente nel giardino, poiché in grado di trattenere gli stranieri (i quali, mangiandone i petali, perderebbero la memoria, dimenticando così la propria patria, cfr. l'episodio dei Lotofagi nel nono libro dell'Odissea), dovrebbe avere un maggior effetto sullo stesso Trebazio, in quanto proprietario della villa.

${ }^{4}$ Conferme che il nome di questo corrispondente sia Fabio e non Fadio si possono trovare in Shackleton Bailey 1962; Demmel 1962: 18, n. 2; White 2010: 33.

${ }^{5}$ In Fam. 9, 25, 2, risalente al marzo del 50, Cicerone, nel raccomandare Fabio Gallo a Lucio Papirio Peto, ne descrive ironicamente l'orientamento filosofico: Cum M. Fabio [...] mibi summus usus est valdeque eum diligo cum propter summam probitatem eius ac singularem modestiam
} 
intestinali, caratterizzati da assenza di febbre ma durati ben dieci giorni. Al fine di riprendersi da questa malattia, l'oratore, rifugiatosi nella sua villa di Tuscolo, si impose un assoluto digiuno di ben due giorni. Causa di tale malessere sarebbe stata una cena a casa di Publio Cornelio Lentulo Spinther ${ }^{6}$, figlio dell'omonimo console del 57 a.C. ${ }^{7}$ e collega di Cicerone nel collegio degli auguri. Questi avrebbe organizzato un banchetto durante il quale avrebbe fatto servire portate a base di funghi, erbe e ortaggi. Secondo quanto riferito da Cicerone, tale scelta di ingredienti sarebbe stata imposta da una recente legge suntuaria, la quale avrebbe ridotto il consumo di pietanze a base di carni e pesce. Questa epistola, pur nella sua linearità e semplicità narrativa, ha sollevato alcuni problemi interpretativi, primo su tutti quello riguardante la datazione. In un contributo del 1993 C. Déry propose di datare l'epistola al 56 a.C. ${ }^{8}$ : la legge suntuaria sarebbe da identificare con la Lex Antia della fine degli anni $70^{\circ}$, mentre la cena coinciderebbe con quella offerta da Lentulo per festeggiare la sua ammissione al collegio degli auguri nel 57 a.C. ${ }^{10}$. La maggior parte degli editori, a partire da Shackleton Bailey ${ }^{11}$, è però concorde nell'abbassare la datazione dell'epistola, facendola risalire a un periodo compreso tra la fine del 46 e l'inizio del 45 tramite un confronto con la restante corrispondenza tra Cicerone e Fabio Gallo (le tre precedenti epistole Fam. 7, 23; 24 e 25) avvenuta tra il dicembre 46 e l'agosto del 45. In questo caso la legge suntuaria sarebbe da identificare con la Lex Iulia sumptuaria emanata da Cesare tra il settembre e il novembre del 46, mentre la cena sarebbe una delle periodiche collegiorum cenae ${ }^{12}$. Questa seconda datazione trova conferma nel gioco ironico che caratterizza l'intera epistola e che ha il suo cardine nelle prescrizioni alimentari volute dalla nuova legge suntuaria di Cesare. Tale provvedimento mirava a riequilibrare le manifesta-

tum quod in iis controversiis quas habeo cum tuis combibonibus Epicuri<i>s optima opera eius uti soleo. Per le attestazioni di stima di Cicerone nei confronti di Gallo cfr. Fam. 2, 14, indirizzata a Marco Celio Rufo e datata approssimativamente al marzo del 50, dove viene descritto come vir optimus e homo doctissimus. Al seguito dell'oratore durante il proconsolato in Cilicia, si occupò anche dell'arredamento di alcune ville dell'Arpinate, cfr. Fam. 7, 23 e Narducci 2003. Medesime attestazioni di stima si ritrovano anche in Fam. 7, 23, 1, indirizzata proprio allo stesso Gallo e risalente al dicembre del 46. Più in generale sul personaggio cfr. Deniaux 1993: 496-498.

${ }^{6}$ Per un profilo generale di Publio Cornelio Lentulo Spinther cfr. Deniaux 1993: 399-401 con relativa bibliografia. Studi successivi in Ryan 1997 e Drummond 2008.

${ }^{7}$ Cfr. Broughton 1952: 199-200; Ryan 2000.

${ }^{8}$ Così Déry 1993.

${ }^{9}$ Ricordata da Macr. 3, 17, 13 e Gel. 2, 24, 13.

${ }^{10}$ Sull'anno della carica cfr. Broughton 1952: 207; sulla cena cfr. la testimonianza di D. C. $39,17,1-2$.

${ }_{11}$ Shackleton Bailey 1977b: 373-374; Cavarzere 2007a: 724-725 e n. introduttiva; Garbarino-Tabacco 2008b: 92-93, n. 1. Così anche Rosivach 2005.

${ }^{12}$ Vedi la testimonianza di Var. R. 3, 2, 16. 
zioni di lusso, fatta eccezione per determinate occasioni e classi di età ${ }^{13}$. Tra i numerosi provvedimenti voluti dalla legge di Cesare, alcuni avevano come scopo quello di misurare gli eccessi della tavola, limitando il consumo di piatti a base di carne e pesce, pietanze molto pregiate, e favorendo così l'uso nei banchetti dei più svariati tipi di verdure e ortaggi, cibi caratterizzati da facile reperimento e pertanto da basso costo $^{14}$. $\mathrm{Al}$ fine di aggirare le restrizioni previste dalla nuova legge di Cesare, i cuochi dell'epoca elaborarono nuove ricette a base di verdure, rese più appetibili con l'aggiunta di salse e intingoli. Così Cic. Fam. 7, 26, 2: Nam dum volunt isti lauti terra nata, quae lege excepta sunt, in honorem adducere, fungos, helvellas, herbas omnis ita condiunt ut nibil possit esse suavius. Sarebbe stato proprio l'uso smodato di queste portate e condimenti ad aver causato i disturbi intestinali di Cicerone. Così continua l'oratore: In eas cum incidissem in cena augurali apud Lentulum, tanta me diárroia arripuit ut hodie primum videatur coepisse consistere. I due cibi incriminati sono elencati subito dopo: Ita ego, qui me ostreis et murenis facile abstinebam, a beta et a malva deceptus sum. Posthac igitur erimus cautiores. L'analisi del passo solleva tuttavia alcuni dubbi: possono tali ortaggi aver effettivamente causato i gravi problemi intestinali di Cicerone? Pietanze a base di bietole e malva sarebbero state in grado di piegare un così ben allenato buongustaio come l'Arpinate? Per capire ciò è bene soffermarsi ulteriormente su questo passo. Il latino beta indica la bietola, nello specifico la beta vulgaris. Tuttavia in questa tipologia rientrano così numerose varietà di bietole ${ }^{15}$ che (causa anche la generica descrizione di Cicerone) risulta molto difficile, se non quasi impossibile, identificare esattamente il tipo di beta consumata dall'oratore. Appare invece più trasparente il termine malva, con il quale si è soliti indicare la Malva sylvestris, pianta erbacea alta fino a un metro dai petali bilobati di colore rosaceo. Sebbene per entrambe le tipologie di piante siano noti effetti lassativi ${ }^{16}$, sembra molto improbabile che un abuso di portate a base di bietole

${ }^{13}$ Così cfr. Suet. Jul. 43: Ius laboriosissime ac severissime dixit. Repetundarum convictos etiam ordine senatorio movit. Diremit nuptias praetorii viri, qui digressam a marito post biduum statim duxerat, quamvis sine probri suspicione. Peregrinarum mercium portoria instituit. Lecticarum usum, item conchyliatae vestis et margaritarum nisi certis personis et aetatibus perque certos dies ademit.

${ }^{14}$ Sui provvedimenti di tale legge cfr. Suet. Jul. 43: Legem praecipue sumptuariam exercuit dispositis circa macellum custodibus, qui obsonia contra vetitum retinerent deportarentque ad se, submissis nonnumquam lictoribus atque militibus, qui, si qua custodes fefellissent, iam adposita e triclinio auferrent. Vedi anche le osservazioni di Rosivach 2005.

${ }^{15}$ Tra cui quelle da orto (Beta vulgaris var. cruenta), da zucchero (Beta vulgaris var. saccharifera), da foraggio (Beta vulgaris var. crassa) o la bietola (Beta vulgaris var. cicla), senza contare le diverse tipologie di tuberi (basti pensare solamente ad alcune varianti quali, per esempio,l'Albina Vereduna).

${ }^{16} \mathrm{Il}$ termine malva deriverebbe dall'espressione latina mollire alveum, chiaro riferimento alle qualità emollienti e lassative della pianta stessa, e sarebbe connesso al greco maláche / malóche, la cui radice mal-compare anche nel verbo malásso, lett. "rendere molle, ammorbidire", e nell'aggettivo malachós. 
e malva abbia potuto causare una diárroia della durata di ben dieci giorni. Qualora si volesse continuare a prendere alla lettera le parole di Cicerone, bisognerebbe allora ipotizzare una diversa causa dei disturbi intestinali, forse riconducibile a un ingerimento eccessivo di alimenti infetti, specie se si tiene presente il livello di igiene e di conservazione dei cibi spesso a quel tempo non adeguato. Ciò nonostante l'assenza di febbre, sintomo che, insieme alla diarrea, spesso si accompagna alla dissenteria infettiva, rende ancora meno credibile l'iperbolica descrizione di Cicerone. Lo stesso Arpinate ci informa tra le righe che anche i suoi i numerosi clientes stentavano a credere all'intensità del suo malessere. Così Cic. Fam. 7, 26,1: Cum decimum iam diem graviter ex intestinis laborarem neque iis qui mea opera uti volebant me probarem non valere quia febrim non haberem, fugi in Tusculanum. Dopo questa lunga premessa si può dedurre come il quadro descritto da Cicerone sia troppo iperbolico per essere ritenuto veritiero. Per quanto sia possibile credere che dopo l'abbuffata a casa di Lentulo l'oratore abbia accusato alcuni dolori intestinali, è tuttavia difficile pensare che tale disturbo sia durato per più di dieci giorni e abbia costretto l'Arpinate a un digiuno quasi totale nella quiete della sua villa di Tuscolo. Si dovrà pertanto ipotizzare che il racconto ciceroniano nasconda un arguto gioco ironico basato sul doppio valore che il cibo ricopre all'interno dell'epistola: l'intero episodio non dovrà essere letto come la clinica descrizione di un malessere fisico, ma come lo spunto per una critica al provvedimento legislativo cesariano. Cicerone si sta infatti rivolgendo a un oppositore del regime di Cesare (Gallo era stato autore di un panegirico di Catone l'Uticense secondo quanto si apprende da fam. 7, $\left.24,2^{17}\right)$, con il quale condivide amore per gli studi e spirito ironico. La cena a base di verdure offerta da Lentulo Spinther offre così l'occasione per una divertente critica alla nuova legge suntuaria. L'intera narrazione del banchetto e l'accenno a funghi, salse, bietole e malve non sono solo semplici descrizioni di un momento conviviale e delle sue conseguenze, ma sono caratterizzati da un valore metaforico, fortemente ironico e quasi grottesco: il divieto di eccedere in carni e pesci duranti i banchetti al posto di portare la sperata litóteta, intesa come austerità e moralità di costumi, avrebbe invece causato solo diárroia a chi l'ha voluta rispettare. L'epistola assume quasi i tratti di una satira romana, genere letterario in cui riferimenti ironici e grotteschi al cibo e al suo valore simbolico sono ricorrenti ${ }^{18}$.

Conferme a questa interpretazione traslata dell'episodio narrato da Cicerone verrebbero anche da alcuni paralleli presenti in altre epistole, più

\footnotetext{
${ }^{17}$ Cic. Fam. 7, 24, 2: "Catonem" tuum mibi mitte; cupio enim legere. Me adbuc non legisse turpe utrique nostrum est.

${ }^{18}$ Importanti osservazioni in Hudson 1989.
} 
o meno contemporanee, in cui il cibo è ancora una volta veicolo di ironia politica nei confronti della legge suntuaria di Cesare. In Fam. 9, 10, 2, datata agli inizi del 45 a.C., l'oratore scrive ironicamente al suo ex-genero Dolabella che si sarebbe aspettato dal suo destinatario una domanda sulla recente cena del grammatico Curzio Nicia di $\operatorname{Cos}^{19}$, durante la vennero offerti dei funghi: Puto te nunc dicere: "oblitusne es igitur fungorum illorum, quos apud Niciam, et ingentium †cularum cum sophia septimae†?". Nonostante la parte finale del periodo presenti insanabili problemi testuali ${ }^{20}$, l'accenno ai funghi rimanda alle nuove mode culinarie escogitate per rispettare le limitazioni imposte dalla legge suntuaria di Cesare ${ }^{21}$. Tale ortaggio, che compariva già in Cic. Fam. 7, 26, 2, si ritrova ancora in Fam. 9, 15, 5, indirizzata da Cicerone all'amico Lucio Papirio Peto e datata alla fine del 46 a.C., anch'essa di poco successiva all'emanazione del provvedimento cesariano: Quid ergo est? Tamen, quamdiu hic erit noster hic praefectus moribus, parebo auctoritati tuae; cum vero aberit, ad fungos me tuos conferam. Domum si habebo, in denos dies singulos sumptuariae legis dies conferam; sin autem minus invenero, quod placeat, decrevi habitare apud te, scio enim me nibil tibi gratius facere posse. Qui la critica alla legge suntuaria è ancora più esplicita. Cicerone, che al momento della scrittura dell'epistola si trovava a Roma, conferma al suo destinatario l'intenzione di restare nell'Urbe fino a quando il praefectus moribus, titolo simile a quello di censore ottenuto da Cesare dopo le vittorie contro i pompeiani in nord Africa ${ }^{22}$, non sarà ripartito dalla capitale. Solo dopo quel giorno l'oratore potrà raggiungere "i funghi di Peto": ad fungos me tuos conferam. Anche in questo caso l'espressione non dovrà essere presa alla lettera, bensì interpretata come un'arguzia. L'accenno ai funghi rimanda ancora una volta alle nuove mode culinarie: Cicerone vuole comunicare all'amico che, appena possibile, lo raggiungerà nella sua casa di Napoli dove le cene e i banchetti saranno a base di funghi, in rispetto alle norme volute da Cesare $^{23}$. Cicerone continua l'ironia nei confronti del provvedimento legislativo affermando che, se troverà una casa in cui abitare a Napoli,

${ }^{19} \mathrm{Su}$ questo grammatico, ricordato da Suet. Gramm. 14, cfr. Groag 1901; per ulteriore bibliografia vedi Shackleton Bailey 1977b, 373.

${ }^{20}$ Corrette le osservazioni di Shackleton Bailey 1977b, 383.

${ }^{21}$ Così già Cavarzere 2007b, 886, 79.

${ }^{22}$ Tale titolo venne conferito a Cesare nel 46 per la durata di tre anni (cfr. D. C. 43, 14, 4), mentre dopo il 44 sarebbe durato per tutta la vita, così Suet. Jul. 76, 1 e D. C. 44, 5, 3; vedi anche Shackleton Bailey 1977b: 352. Tuttavia non si deve escludere che nelle parole di Cicerone si nasconda una certa ironia nei confronti di questa particolare carica politica.

${ }^{23}$ Così già Cavarzere 2007b: 907 n. 126. 
provvederà ad applicare per dieci giorni ${ }^{24}$ quanto la legge prescrive per un solo giorno: altra iperbole, altro scherzo ${ }^{25}$.

\section{II) Pavoni in brodo.}

Un caso analogo di ironia politica veicolata tramite un riferimento al mondo culinario è presente in un'altra lettera indirizzata a Lucio Papirio Peto. In Cic. Fam. 9, 18, risalente al luglio del 46, Cicerone si rallegra che l'amico abbia approvato la sua decisione di intraprendere la carriera di maestro di retorica. Dal Cic. Fam. 9, 16, $7^{26}$ veniamo a sapere che gli allievi dell'oratore erano due cesariani, rispettivamente Aulo Irzio, l'autore dell'ottavo libro del De bello Gallico, e il già citato Publio Cornelio Dolabella: Cicerone, l'antico sostenitore del partito pompeiano, si trovava a essere maestro proprio di due tra i cesariani più in vista. L'Arpinate, elencando a Peto i vantaggi della propria scelta, si serve di un'immagine tratta dal mondo culinario: Extremum illud est, quod tu nescio an primum putes: plures iam pavones confeci, quam tu pullos columbinos; tu istic te Hateriano iure delectas, ego me hic Hirtiano. Cicerone sta giocando con i doppi sensi: I) il termine pavo veniva usato tanto come "pavone" (animale allora molto pregiato e costoso ${ }^{27}$ ) quanto per indicare un simbolo di vanità 28 ;I) il verbo conficio è traducibile sia con il significato di "mangiare, masticare"29 sia con quello di "consumare, annientare" ${ }^{30}$; III) pullus indica il "piccolo gallo", soprattutto un volatile ancora in età da nido ${ }^{31}$; tale

${ }^{24} \mathrm{Si}$ noti il dato temporale denos dies: dieci giorni era anche la durata dei disturbi intestinali di Cicerone riportata apertura di Cic. fam. 7, 26, 1: Cum decimum iam diem. La somiglianza delle due cronologie (entrambe presenti all'interno di una critica alla legge suntuaria) potrebbe indurre a pensare che si tratti di cifre canoniche. Ciò avvalorerebbe ancora di più l'idea che l'iperbolica durata e intensità dei malesseri di Ciceroni altro non sarebbero che un espediente letterario.

${ }^{25} \mathrm{La}$ frase potrebbe essere interpretata anche come un accenno alla bassa severità o allo scarso controllo messo in pratica dalla legge di Cesare, cfr. Rosivach 2005 e l'osservazione di Cavarzere 2007b: 907, n. 127.

${ }^{26}$ Spedita da Tuscolo e datata alla metà del luglio 46.

${ }^{27}$ Per uno studio del pavone nel mondo romano e nelle fonti latine cfr. Capponi 1979: 389-393, il quale a p. 392, per spiegare l'alto valore economico di questo volatile, rimanda a Col. 8, 11, 1: Pavonum educatio magis patrisfamilias quam tetrici rustici curam poscit. Cfr. anche Var. $R .3,6,1-5$; vedi in particolare $R .3,6,6$, dove Varrone precisa che Quinto Ortensio per primo fece imbandire il pavone durante una cena per festeggiare l'inizio della sua edilità, segnando così l'inizio di una moda costosa. Sul significato concreto di pavo cfr. TLL X 1 col. 386, 10-15.

${ }^{28}$ Forse derivato dalle teorie sull'indole dei pavoni, ritenuta orgogliosa per via del loro folto e colorato piumaggio, cfr. Plin. Nat. 10, 43. Vedi anche TLL X 1 col. 836, 67-70.

${ }^{29}$ Sinonimo di mandere, comedere, consumere, cfr. TLL IV col. 201, 62-72; col valore di digerire cfr. TLL IV 201, 73-202, 2.

${ }^{30} \mathrm{Col}$ significato di debilitare, cruciare cfr. TLL IV col. 202, 3-35; perdere cfr. 203, 54-204, 15; delere, extinguere, absumere cfr. 204, 47-65.

${ }^{31}$ Cfr. Capponi 1979: 435. 
significato è parzialmente ripreso dall'aggettivo columbinus, usato per definire gli uccelli in grado di nidificare dopo cinque o sei mesi ${ }^{32}$; IV) ius è omografo sia del termine indicante il "diritto", derivato dalla radice *ieuos/*iouos ${ }^{33}$, sia di quello per "brodo, succo", dalla radice *ius ${ }^{34}$. Qualora si traducesse il passo ciceroniano conferendo a ogni parola presa in esame un significato concreto, vicino al lessico culinario, si otterrebbe la seguente traduzione: "Questo è l'ultimo vantaggio, che credo tu metterai al primo posto: ho già mangiato più pavoni io che piccoli galletti tu. Lì tu gusti il brodo di Aterio, qui io quello di Irzio". Secondo questa interpretazione, Cicerone sembrerebbe comunicare all'amico che la sua attività di maestro di retorica gli frutterebbe molti vantaggi economici: gli allievi Irzio e Dolabella lo ricompenserebbero così profumatamente da permettergli di cenare con costosissimi e pregiati pavoni, cotti nel brodo di Irzio, a differenza di Peto, che con la sua normale attività di giurista si potrebbe permettere solo cene a base di piccoli galletti, cotti nel brodo delle cene col giurista Aterio ${ }^{35}$. Tuttavia, qualora si conferisse a ogni termine un valore traslato, l'intero periodo assumerebbe un valore diverso, ironico quanto arguto: "Questo è l'ultimo vantaggio, che credo tu metterai al primo posto: io ho già spennato più uomini che si pavoneggiano che piccoli galletti tu. Lì tu degusti il diritto di Aterio, qui io il brodo di Irzio". Ancora una volta l'immagine che ne deriva sembra assumere tinte da satira o da commedia plautina: Cicerone, astuto maestro di retorica dai trascorsi pompeiani, avrebbe come allievi Irzio e Dolabella, cesariani resi vanitosi dalla vittoria della fazione di appartenenza; quest'ultimi si comporterebbero proprio come due pavoni che sguazzano nel brodo preparato da un cuoco sopraffino, ignari di essere cotti poco a poco. I ruoli politici sono invertiti, dal momento che non è più il pompeiano a temere $i$ due cesariani, ma sono invece i due cesariani a fare inconsapevolmente il gioco del pompeiano. La metafora che unisce il mondo politico con quello culinario, incentrata sul doppio valore dei termini usati, si basa sulla condivisione di un bagaglio culturale, lessicale e ideologico comune a Cicerone e Peto. A ciò si aggiunga che il gioco di parole richiama forse alla memoria dei due corrispondenti lo ius Verrinum citato in

${ }^{32}$ Cfr. Capponi 1979: 185-186.

${ }^{33}$ Connesso al Sanscrito yoh, cfr. Walde-Hofmann 1938a: 733-734; Pokorny 1959: 512. Gli autori latini ne spiegavano l'origine a partire da iustum/iustitia, cfr. Isid. Orig. 5, 3, 1: ius [...] dictum quia iustum est, così Maltby 1991: 319.

34 Connesso al Sanscrito yus, cfr. Walde-Hofmann 1938a: 734; Pokorny 1959: 507. Maltby 1991: 319 evidenzia che il termine veniva sentito affine ora all'aggettivo iucundus (cfr. Var. L. 5, 109: Elixum e liquore aquae dictum; et ex iure, quod iucundum magis conditione) ora, quasi ironicamente, al lessico giuridico (cfr. Isid. Orig. 20, 2, 32: ius coquinae magistri a iure nuncupaverunt, quia [ea] est lex condimenti eius).

${ }_{35}$ Personaggio di non semplice identificazione: secondo Shackleton Bailey 1977b: 341 si trattava di un giurista o di un vicino di casa o di un ospite di Peto; Sumner 1983: 174 ritiene che possa trattarsi del padre oppure dello zio del famoso oratore Quinto Aterio. 
Cic. Ver. 1, 121, in un attacco fortemente sarcastico nei confronti di Verre ${ }^{36}$. Infine non bisogna neanche escludere che parte dell'ironia dell'episodio sia da ricercare nello stesso ius, inteso come brodo o salsa, la cui ironica ripresa in Cic. fam. 9, 20, 2 suggerisce che tale pietanza era probabilmente ritenuta da Peto come l'orgoglio della propria tavola ${ }^{37}$.

\section{III) Da cavalli a ronzini: la memoria delle cene passate di Peto.}

Dall'inizio della lunga Fam. 9,16 $6^{38}$ apprendiamo che, in una precedente lettera ora perduta, Peto aveva manifestato a Cicerone la sua preoccupazione per le possibili conseguenze politiche di alcune battute anticesariane attribuite all'oratore e riportate al dittatore ${ }^{39}$. L'Arpinate, dopo aver tranquillizzato il proprio corrispondente lodando la finissima capacità di giudizio di Cesare e dimostrando la giusta cautela del proprio agire ${ }^{40}$, si concentra nuovamente sulla lettera dell'amico. Peto infatti, nella seconda parte della sua missiva, doveva aver descritto un pasto frugale offerto da un certo Popilio ${ }^{41}$, la cui portata principale era una torta al formaggio e pesce. Così Cic. Fam. 9, 16, 7: Nunc venio ad iocationes tuas, quoniam tu secundum Oenomaum Attii, non, ut olim solebat, Atellanam, sed, ut nunc fit, mimum introduxisti. Quem tu mibi pompilum, quem denarium narras? Quam tyrotarichi patinam?. Successivamente Cicerone, dopo aver elencato i lussi che gli deriverebbero dall'insegnare retorica a Irzio e Dolabella ${ }^{42}$, ironizza sulle continue lamentele di Peto circa la propria insolvenza. Questa era stata causata durante il 47 dal tentativo di Cesare di risolvere il complesso problema dei debiti: il dittatore aveva imposto una liquidazione forzosa dei debiti di coloro che erano in possesso di terreni; tale provvedimento, parallelo al crollo del prezzo dei beni immobili, fece in modo che molti creditori si ritrovassero in carenza di liquidità ${ }^{43}$.

${ }^{36}$ Cfr. Mitchell 1986: 212.

${ }^{37}$ Vedi Shackleton Bailey 1977b: 341 e 346.

${ }^{38}$ La lettera, datata alla metà del luglio 46, venne inviata probabilmente dalla villa di Tuscolo.

${ }^{39}$ Così Shackleton Bailey 1977b, 335.

${ }^{40}$ Cfr. le osservazioni di Hall 2009: 83.

${ }^{41}$ Popilius è forma tramandata dai codici. Demmel 1962: 53, n. 1 ha proposto l'emendazione Po $<m>$ pilius, suggerendo così di identificare l'ospite di Peto con il grammatico di origine siriaca Marco Pompilio Andronico, ricordato da Suet. Gramm. 8 (cfr. il commento di Kaster 1995: 122-128, che però non accenna all'epistola ciceroniana), il quale avrebbe trascorso gli ultimi anni a Cuma inops atque egens. L'ipotesi è stata seguita da Shackleton Bailey 1977b: 337, ma cfr. Cavarzere 2007b: 914-915, secondo il quale tale idea, sebbene affascinante, non è necessaria, in quanto la forma tramandata dai codici rispecchia un gentilizio molto diffuso in area campana.

${ }^{42}$ Cic. Fam. 9, 16, 7: Hirtium ego et Dolabellam dicendi discipulos habeo, coenandi magistros; puto enim te audisse, si forte ad vos omnia perferuntur, illos apud me declamitare, me apud illos coenitare. Vedi anche il secondo paragrafo della presente analisi.

${ }^{43}$ Cfr. Shackleton Bailey 1966: 377 e 1977b: 338 che rimanda a Frederiksen 1966: 133; vedi anche Cavarzere 2007b: 916, n. 147. 
Peto, sebbene stretto in questa condizione, doveva aver proposto a Cicerone di accoglierlo come ospite nella sua casa, annunciandogli però di non essere in grado di proporgli niente altro che pasti frugali come quelli preparati da Matre di Tebe, autore di inni famoso per la parsimonia dei propri costu$\mathrm{mi}^{44}$. L'oratore ironizza così sulle semplici portate che Peto doveva avergli descritto. Cfr. Cic. Fam. 9, 16, 8: Volo enim videre animum, qui mibi audeat ista, quae scribis, apponere aut etiam polypum miniati Iovis similem. Mibi crede, non audebis: ante meum adventum fama ad te de mea nova lautitia veniet; eam extimesces. Neque est, quod in promulside spei ponas aliquid, quam totam sustuli; solebam enim antea debilitari oleis et lucanicis tuis. Cicerone non desidera cene o pranzi dalle portate illimitate, ma pasti raffinati e ricercati, proprio come il suo palato: nessuno avrebbe il coraggio di servirgli semplici polpi cotti e dal colore rosso ${ }^{45}$ come la statua in terracotta di Giove Capitolino (la quale era periodicamente riverniciata con il minio, una polvere dal colore rosso-arancione ${ }^{46}$ ); parimenti inutile per Peto sarebbe il preparare numerosi antipasti a base di olive e salsicce per saziare il commensale prima delle vere e proprie portate. Il passo non deve essere letto come una presa in giro delle scarse finanze dell'amico o come un attacco nei confronti dell'avarizia di Peto. Lo stesso Cicerone aggiunge nel paragrafo successivo che quanto da lui scritto è un puro e semplice scherzo: un'amichevole accoglienza e un bagno caldo saranno più che sufficienti. Tra le righe dell'arguto gioco ciceroniano si cela una velata ironia nei confronti del provvedimento voluto da Cesare per la soluzione del problema dei debiti: la conseguenza non sarebbe stata un miglior equilibrio sociale, bensì la scomparsa della liquidità dei creditori. La miseria della tavola, ridotta a un semplice polpo cotto in acqua, è metafora della penuria di risultati che caratterizza la legge di Cesare. Lo scherzo torna nella già citata Cic. Fam. 9, 18, 4: Si aestimationes tuas vendere non potes neque ollam denariorum implere, Romam tibi remigrandum est: satius est hic cruditate, quam istic fame. Ancora una volta la frugalità della tavola è presa come spunto per lamentare gli effetti del provvedimento legislativo. Qui l'ironia è resa ancora più evidente dalla vicina presa in giro dei superbi Irzio e Dolabella ${ }^{47}$.

${ }^{44}$ Per un primo studio complessivo di Matre di Tebe cfr. Hobein 1930.

${ }^{45}$ Secondo Shackleton Bailey 1977b: 338 si trattava probabilmente di un polpo cotto in una salsa rossa; più correttamente Cavarzere 2007b: 917, n. 151 (che rimanda a Della Valle 1933: 158 s.), il quale lo identifica con un piatto della cucina popolare che farebbe riferimento al colore del polipo dopo la cottura nella sua stessa acqua. Sulla forma miniati preferita alla variante ricinati proposta da Reich 1903: 62-63, n. 5, cfr. le giuste argomentazioni di Shackleton Bailey 1977b: 338-339.

${ }^{46}$ Cfr. le testimonianze di Plin. Nat. 33, 111 e Plu. Quest. Rom. 98; in Plin. Nat. 35, 157 Plinio ritiene che veniva verniciata con il colore rosso del minio in quanto fabbricata in terracotta, ma cfr. Serv. Buc. 10, 27 afferma che il rosso è il colore dell'etere e che Giove sarebbe lui stesso l'etere (cfr. anche le osservazioni di Zehnacker 1983: 199-200).

${ }^{47} \mathrm{C}$ fr. il secondo capitolo della presente analisi. 
Cicerone ribadisce il gioco poco dopo con un'altra immagine tratta dal mondo della cucina: Potes mulo isto, quem tibi reliquum dicis esse, quoniam cantherium comedisti, Romam pervehi. La battuta, che allude al proverbio "passare da asini a cavalli", indicante un repentino rovescio di fortuna ${ }^{48}$, si colora di un ulteriore tratto ironico se si pensa che dalle tavole greche e romane era solitamente assente la carne equina ${ }^{49}$. Un ultimo riferimento si ritrova infine in Cic. Fam. 9, 20, 1, spedita a Peto da Roma all'inizio dell'agosto 46: Illud doleo, in ista loca venire me, ut constitueram, non potuisse; babuisses enim non hospitem, sed contubernalem. At quem virum! non eum, quem tu es solitus promulside conficere: integram famem ad ovum affero, itaque usque ad assum vitulinum opera perducitur. Anche qui tornano gli elementi già riscontrati in precedenza. Cicerone ironizza sul proprio palato sopraffino, affermando di non essere un commensale qualunque che può essere soddisfatto con un antipasto: neppure le uova (ritenute parte conclusiva dell'antipasto, ma qui considerate come prima portata della cena ${ }^{50}$ ) potrebbero fiaccare un allenato buongustaio, il cui appetito giungerebbe intatto fino al vitello arrosto, l'ultima portata prima del dolce ${ }^{51}$. La fame non è più saziata dalla lotta per lo stato e dall'attività in tribunale: la situazione politica è tale che agli uomini come Cicerone non resta che abbandonare il foro in favore dello studio della filosofia e delle cene con gli amici: Nunc omnem nostram de re publica curam, cogitationem de dicenda in senatu sententia, commentationem causarum abiecimus, in Epicuri nos adversarii nostri castra coniecimus, nec tamen ad hanc insolentiam sed ad illam tuam lautitiam, veterem dico, cum in sumptum habebas, etsi numquam plura praedia habuisti. Cicerone continua a canzonare il proprio corrispondente: la cena non dovrebbe essere caratterizzata dall'eccesso nella quantità delle portate, ma dalla ricercatezza delle stesse, proprio come era solito fare Peto prima di subire gli effetti della mancanza di liquidità ${ }^{52}$. Ancora una volta la scarsa raffinatezza della tavola imbandita riflette la bassa qualità del provvedimento cesariano. La frugalità del banchetto di Peto funge da tramite per ironizzare nei confronti della politica del dittatore: ciò è ulteriormente ribadito sia dal tono di delusione politica che caratterizza l'inizio e la fine dell'epistola sia dalla rinnovata presa in giro di Irzio.

\footnotetext{
${ }^{48}$ Vedi Otto 1890: 233, ma soprattutto Tosi 1991: 357-358, numero 762.

${ }^{49}$ Cfr. Curt. 9, 10, 12.

${ }^{50}$ Cfr. le osservazioni di Shackleton Bailey 1977b: 344-345.

${ }^{51}$ Vedi ancora Shackleton Bailey 1977b: 345.

${ }^{52} \mathrm{Cfr}$. ancora Cic. Fam. 9, 20, 2: dediscendae tibi sunt sportellae et artolagyni tui.
} 


\section{IV) Cene mancate e arte arus picina: un CASo PARTicolare.}

Una conferma del tono ironico e della complicità intellettuale che caratterizza il carteggio tra Cicerone e Peto giunge da Cic. Fam. 9, 24, datata al gennaio o ai primi di febbraio del 43. L'oratore, rispondendo a una lettera di raccomandazione in favore di un non meglio identificato Rufo, si dispiace che il suo destinatario abbia preso la decisione di non frequentare più $i$ banchetti degli amici. L'Arpinate ironizza su questa scelta: se Peto ha fatto pochi progressi nell'arte culinaria quando poteva imitare le cene a cui era invitato, ora, non frequentandole più, sarà destinato a disimparare anche il poco che già sapeva. Così Cic. Fam. 9, 24, 2: Te ad coenas itare desisse moleste fero; magna enim te delectatione et voluptate privasti; deinde etiam vereor-licet enim verum dicere-, ne nescio quid illud, quod solebas, dediscas et obliviscare coenulas facere; nam, si tum, cum habebas, quos imitarere, non multum proficiebas, quid nunc te facturum putem?. L'Arpinate continua la sua arguzia affermando che la decisione di Peto non di frequentare più i banchetti sarebbe così preoccupante da richiedere l'intervento dell'arte aruspicina: Spurinna quidem, cum ei rem demonstrassem et vitam tuam superiorem exposuissem, magnum periculum summae rei publicae demonstrabat, nisi ad superiorem consuetudinem tum, cum Favonius flaret, revertisses; hoc tempore ferri posse, si forte tu frigus ferre non posses. L'episodio è creato ad arte. Spurinna, l'aruspice che, secondo la tradizione ${ }^{53}$, aveva intimato a Cesare di non recarsi in senato il giorno delle idi di marzo, avrebbe risposto a Cicerone che Peto, continuando a non frequentare i pranzi e le cene, esporrebbe a un grave pericolo l'intero stato. L'ironia del passo è causata dalla parodia del lessico politico-forense nella formula magnum periculum sommae rei publica $e^{54}$ e dal rovesciamento comico della figura di Spurinna: l'anno precedente aveva predetto a Cesare di non recarsi al senato e non uscire di casa per avere salva la propria vita; ora invece consiglia a Peto di tornare a cenare fuori casa per salvare le sorti dello stato ${ }^{55}$. Qui una cena non consumata è il tramite per creare un pungente gioco ironico sia verso il ricordo e l'eredità politica di Cesare sia nei confronti dell'aruspicina e di uno dei suoi più famosi esponenti ${ }^{56}$.

${ }^{53}$ Cfr. Suet. Jul. 81, 2-4; V. Max. 8, 11, 2. Ma cfr. Vell. 2, 57, 2; Plu. Caes. 63; D. C. 44, 18, 4; App. $B C 2,149$, dove non è precisato il nome dell'aruspice. Nessun accenno alla sua previsione in Verg. G. 466-488.

${ }^{54}$ Cfr. TLL X 1 col. 1462, 10-38.

${ }^{55}$ La cifra caricaturale è colta anche da Pease 1973: 311.

${ }^{56}$ Cicerone non era nuovo a questo tipo di accuse. In Cic. Fam. 6, 18, 1, datata al gennaio del 45 e indirizzata a Quinto Paconio Lepta, l'oratore rivolge un pungente attacco nei confronti degli aruspici ancora in attività e ammessi da Cesare in senato: neque enim erat ferendum, cum, qui hodie haruspicinam facerent, in senatum Romae legerentur, eos, qui aliquando praeconium fecissent, in municipiis decuriones esse non licere. Per un commento del passo cfr. Shackleton Bailey 1977b: 383-384. Sull'episodio cfr. Rawson 1978: 143-145 e Zecchini 2001: 68-69. 


\section{Conclusioni}

L'ironia nell'opera ciceroniana è stata ampiamente studiata sia in relazione al suo valore retorico sia come tratto distintivo di numerose epistole ${ }^{57}$. Dai passi qui analizzati emerge un particolare uso dell'ironia legato alla descrizione di cibi e banchetti. Tali particolari arguzie, ora esplicite ora alquanto criptiche ${ }^{58}$, sono solitamente racchiuse all'interno di brevi quadri narrativi, costruiti quasi come episodi in sé conclusi e facilmente separabili dal resto dell'epistola nella quale sono tramandati. In queste particolari sezioni, pietanze, semplici ingredienti o più elaborate ricette diventano strumenti retorici finalizzati a ironizzare sui propri avversari. Per ottenere ciò Cicerone si serve di tutte le armi messe a disposizione dalla retorica, quali metafore, similitudini, parodie, allusioni. Si deduce pertanto che la comprensione del duplice significato con cui vengono caricate le immagini culinarie avviene tramite la condivisione di un medesimo codice culturale tra mittente e destinatario. Il cibo, grazie al suo legame con la quotidianità, e per questo spesso presente nei generi letterari della satira e della commedia, diviene il tramite preferenziale per divertire. L'ironia che ne scaturisce, sebbene a una prima lettura sembri legata più allo stomaco che alla mente, è in realtà molto sottile: gli episodi creati da Cicerone sono lontani dalla bassa trivialità o dalla comicità fine a se stessa, la quale cerca il riso solo nella situazione paradossale e nel linguaggio grottesco. Le scene narrate dall'oratore non si spiegherebbero e non sarebbero comprensibili senza una basilare conoscenza di nozioni filosofiche ${ }^{59}$ (si pensi agli accenni a Epicuro presenti in Fam. 7, $26)^{60}$ e senza un preciso aggancio alla realtà politica contemporanea ai corrispondenti. I singoli cibi citati perdono il loro valore concreto e diventano metafore con cui Cicerone si prende gioco degli avversari e con cui filtra la tragica realtà politica per mezzo del riso. Allusioni, lessico greco, proverbi e giochi di parole presuppongono un medesimo codice culturale tra mittente e destinatario necessario per comprendere ogni immagine: gli effetti della malva e della bietola descrivono gli effetti della nuova legge suntuaria; la costante presenza di funghi sulle tavole ironizza sulle imposte limitazioni alimentari; i superbi Irzio e Dolabella non sono altro che pavoni cotti in brodo; le scarse portate della tavola di Peto riflettono la scarsa qualità della risoluzione dei debiti adottata da Cesare. Risulta pertanto chiaro che i singoli

\footnotetext{
${ }^{57}$ Sull'ironia nell'opera ciceroniana cfr. il fondamentale, sebbene orami datato, volume di Haury 1955; sul sarcasmo nelle ad familiares cfr. Hutchinson 1998: 172-199; Chessa 1999: 247-250; Schneider 2000.

${ }^{58}$ Sulla riservatezza nelle lettere ciceroniane cfr. Nicholson 1994-1995; Cavarzere 1998: 12-17; Chessa 1999: 243-246.

${ }^{59}$ Sulla filosofia nelle lettere ciceroniane cfr. il recente volume di McConnell 2014.

${ }^{60} \mathrm{Cfr}$. Rosivach 2005.
} 
cibi descritti non sono elementi descrittivi scelti a caso, ma veri e propri elementi narrativi ricercati da Cicerone per una particolare caratteristica, per la loro scarsa o alta qualità e per un duplice valore, prima concreto poi astratto, legato al termine stesso: la realtà materiale del singolo alimento è così trasformata in arguta ironia politica.

\section{Bibliografia}

Broughton, T. R. S (1952), The Magistrates of Roman Republic, Vol. II, 99 B.C. - 31 B.C., New York.

Capponi, F. (1979), Ornithologia latina, Genova.

Cavarzere, A. (2007), "Libro VII. Introduzione, traduzione e note", in A. Cavarzere (coord.), Lettere ai Familiari, vol. I, introduzione a cura di E. Narducci, Milano, 637-747.

Cavarzere, A. (2007), "Libro IX. Introduzione, traduzione e note", in A. Cavarzere (coord.), Lettere ai Familiari, vol. II, introduzione a cura di E. Narducci, Milano, 845-957.

Cavarzere, A. (1998), "Caro amico ti scrivo", in A. Chemello (coord.), Alla lettera. Teorie e pratiche epistolari dai Greci al Novecento, Milano, 11-33.

Chessa, A. (1999), "Aspetti di espressività nell'epistolario ciceroniano", AFLC 17: 205253.

Della Valle, G. (1933), Tito Lucrezio Caro e l'epicureismo campano, Napoli.

Demmel, M. (1962), Cicero und Paetus (Ad fam. IX,15-26), Köln.

Deniaux, É. (1993), Clientèles et pouvoir à l'époque de Cicéron, Roma.

C. Déry, The Lex sumptuaria in Cicero, Ad familiares 7.26, LCM 18, 1993: 156-157.

Drummond, A. (2008), "The Ban on «gentiles» Holding the Same Priesthood and Sulla's Augurate", Historia 57 (4): 367-407.

Frederiksen, M.W. (1966), "Caesar, Cicero and the Problem of Debt”, JRS 56: 128-141.

Garbarino, G., Tabacco, R. (2008a), M. Tullio Cicerone, Ad familiares, Vol. I, Torino.

Garbarino, G., Tabacco, R. (2008b), M. Tullio Cicerone, Ad familiares, Vol. II, Torino.

Groag, E (1901), "Curtius Nicias (22)", in A. F. Pauly, G. Wissowa (coord.), Paulys Realencyclopädie der classischen Altertumswissenschaft, Bd. IV 2, Stuttgart, 18681869.

Hall, J. (2009), Politeness and Politics in Cicero's Letters, Oxford.

Haury, A. (1955), L'ironie et l'humour chez Cicéron, Leiden.

Hobein, H. (1930), "Matris", in F. Pauly, G. Wissowa, W. Kroll (coord.), Paulys Realencyclopädie der classischen Altertumswissenschaft, Bd. XIV 2, Stuttgart, 22872298.

Hudson, N. A. (1989), "Food in Roman Satire", in S. H. Braund (ed.), Satire and society in Ancient Rome, Exeter, 69-87.

Hucthinson, G. O. (1998), Cicero's Correspondence. A literary Study, Oxford. 
Kaster, R. A. (1995), C. Svetonius Tranquillus, De grammaticis et rhetoribus, Oxford.

Maltby, R. (1991, $2^{\text {nd }}$ ed.), A Lexicon of Ancient Latin Etymologies, Leeds.

McConnell, S. (2014), Philosophical Life in Cicero's Letters, Cambridge.

Mitchell, T. N. (1986), Cicero, Verrines II.1, Warminster.

Narducci, E. (2003), “La memoria della grecità nell'immaginario delle ville ciceroniane”, in M. Citroni (coord.), Memoria e identità. La cultura romana costruisce la sua immagine, Firenze, 119-148.

Nicholson, J. (1994-1995), “The Delivery and Confidentiality of Cicero's Letters”, CJ 90: 33-63.

Otto, A. (1890), Die Sprichwörter und sprichwörtlichen Redensarten der Römer, Leipzig.

Pease, A. S. (1973), M. Tulli Ciceronis de divinatione libri duo, Darmstadt.

Préchac, F. (1913), “Notes on the Trebatius the Velian”, CQ 7: 273-281.

Rawson, E. D. (1978), “Caesar, Etruria and the Disciplina Etrusca”, JRS 68, 32-52.

Reich, H. (1903), Der Mimus. Ein Litterar-entwickelungsgeschichtlicher Versuch, Erster Band, Erster Teil, Theorie des Mimus, Berlin.

Rosivach, V.J. (2005), “Cicero, Epistulae ad familiares 7.26 (Why Cicero Got Sick from Eating Too Many Veggies)”, NECJ 32 (4): 319-327.

Ryan, F. X. (1997), "The Birth-dates of Domitius and Scipio”, AHB 11 (2-3): 89-93.

Ryan, F. X. (2000), “The Praetorship of P. Cornelius Lentulus Spinther”, Hermes 128 (2): 246-247.

Schneider, W. C. (2000), "Vom Salz Ciceros: zum politischen Witz, Schmäh und Sprachspiel bei Cicero", Gymnasium 107: 497-518.

Shackleton Bailey, D. R. (1962), “Two Tribunes, 57 B.C.”, CR 12 n.s.: 195-196.

Shackleton Bailey, D. R. (1966), Cicero's Letters to Atticus, Vol. V, Cambridge.

Shackleton Bailey, D. R. (1977), Epistulae ad Familiares, vol. I, 62-47 a.C., Cambridge.

Shackleton Bailey, D. R. (1977), Epistulae ad familiares, vol. II, 47-43 B.C., Cambridge.

Sumner, G. V. (1983), “D. R. Shackleton Bailey, Cicero: Select Letters. Review”, CPh 78: $173-175$.

Tabacco, R. (2009), “A cena coi potenti. Cibo, convivialità e potere nelle lettere di Cicerone”, in G. Tesio, G. Pennaroli (coords.), Di cotte e di crude. Cibo, culture, comunità. Atti del Convegno internazionale di studi, Vercelli - Pollenzo, 15-16-17 marzo 2007, Torino, 27-51.

Tosi, R. (1991), Dizionario delle sentenze latine e greche, Milano.

Walde, A. (1938, $3^{\text {rd }}$ ed.), Lateinisches Etymologisches Wörterbuch, 3., neubearbeitete Auflage von J. B. Hofmann, erster Band A - L, Heidelberg.

White, P. (2010), Cicero in Letters. Epistolary Relations of the Late Republic, Oxford.

Zecchini, G. (2001), Cesare e il mos maiorum, Stuttgart.

Zehnacker, H. (1983), Pline l'Ancien, Histoire Naturelle, Livre XXXIII, Paris. 